Historic, Archive Document

Do not assume content reflects current scientific knowledge, policies, or practices. 


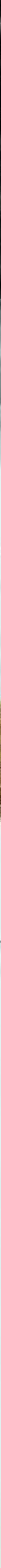
Incorporated

Farm:

Preston Street Road at Audubon Park
Louisville, Kentucky

P. O. Address

R. 3 Box 258

\section{Price List of up-to-date Dahlias}

We send out strong field grown tubers. Will furnish plants at half the tuber price. Unless previously arranged for all orders will be sent C. O. D.

A. C. LAWRENCE. Hybrid Cactus. Each

Flesh pink, large and fine. $\$ 1.00$

AGNES HAVILAND. Decorative.

Rosy pink, suffused yellow.

AL KORAN. Decorative.

Goldenrod and amber.

ALEX. WALDIE. Hybrid Cactus.

Creamy yellow overlaid pink.

AMUN RA. Decorative.

Coppery bronze, shading to gold and amber............. 1.50

ALVA CAMPBELL. Peony.

Flame red. Very large....

AMBASSADOR. Hybrid Cactus.

Solt yellow with amber and pink shadings.

AMERICAN BEAUTY. Show.

Crimson with purple suffusion.

ATTRACTION. Hybrid Cactus.

Lavender. Fine for cutting.

BALLET GIRL. Cactus.

Orange edged white........................ 1.00

BASHFUL GIANT, Decorative.

Dull apricot shaded gold.

BEN WILSON. Decorative.

Red with gold tips......................... 1.00

BERTHA JOST. Decorative.

Soft yellow with pink suffusion

BONNIE BRAE. Decorative.

Cream shaded blush pink...................... 1.00

BREAK O'DAY. Cactus.

Sulphur yellow............................... .50

CAL. SUPERBA. Decorative.

Light pink .............................. 2.00

CARDINAL MERCIER. Peony.

Flesh pink veined canary ...................... 1.50

CATHERINE WILCOX. Decorative.

White over-laid rosy violet ...................... 1.00

CHAMPAGNE. Decorative.

Dull gold changing to chamois.................... 2.50

CHARM. Decorative.

Burnt orange............................... 1.00

CIGARETTE. Hybrid Cactus.

Creamy white, heavily edged red $\ldots \ldots \ldots \ldots \ldots \ldots \ldots .1 .50$

C. H. JAMES. Peony.

Waxy light cream clouded cerise $\ldots \ldots \ldots \ldots \ldots \ldots \ldots \ldots . \quad .50$

CLEMENCEAU. Cactus.

Bright yellow tipped white..................... 2.50

COUNTESS OF LONSDALE. Cactus.

Salmon pink, free.............................. $\quad .25$

DAKOTA. Decorative.

Pleasing shade of brownish red.................. 1.00

DIANA. Peony.

Dark claret with long pointed petals............... .50 
DON WILLIAMS. Decorative.

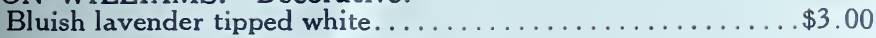

DR. TEVIS. Decorative.

Salmon rose suffused gold ..................... 50

EARL WILLIAMS, Decorative.

Brilliant crimson and white-well distributed......... 1.50

ECHO. Decorative.

Empire yellow shaded salmon. Fine.............. 1.50

ED. LACHAM. Hybrid Cactus.

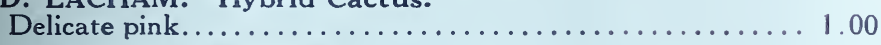

ELDORADO. Decorative.

Pure gold. Large and fine................... 3.00

ELENA EYRE. Decorative.

Amaranth pink............................ 2.00

EL JEBEL. Hybrid Cactus.

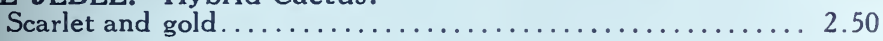

ELSIE OLIVER. Hybrid Cactus.

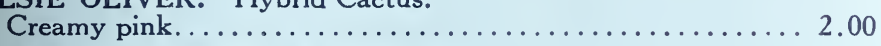

EMMA SLOCOMBE. Decorative.

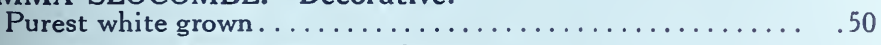

ESTHER HOLMES. Hybrid Cactus.

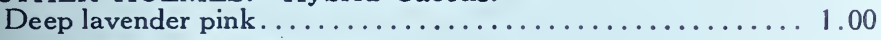

E. T. BEDFORD. Decorative.

Silvery purple. Immense................. 00

ETENDARD DE LYON. Cactus.

Rich carmine rose...................... 50

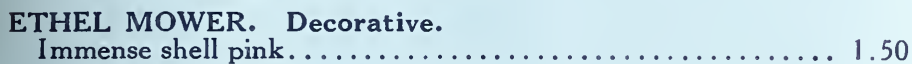

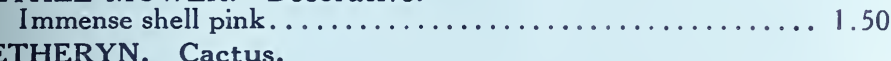

Rose pink............................... 2.00

FRANCIS CATHERINE. Decorative.

Light orange ....................... 5.00

FRANCIS LOBDELL. Hybrid Cactus.

Mallow pink shading to white center. Very large........ 2.00

GEISHA. Peony.

Red, gold and scarlet..................... 50

GEORGE WALTERS. Hybrid Cactus.

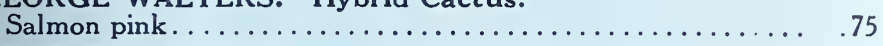

GENEVEVA. Cactus.

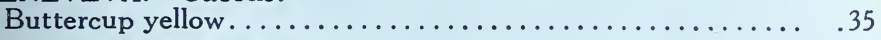

GLADYS BATES. Cactus.

Tan, reverse rose. ......................... 00

GLADYS SHERWOOD. Hybrid Cactus.

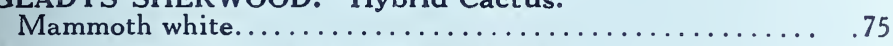

GLORY OF NEW HAVEN. Decorative.

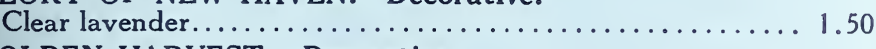

GOLDEN HARVEST. Decorative.

Immense yellow stripped carmine............. 1.50

GOLDEN WEST. Cactus.

Old gold.............................. 50

GRACE WIGGINTON. Peony.

White overlaid rosy carmine ................. 50

GRETCHEN. Peony.

Pure white tipped cerise . . . . . . . . . . . . . . . .

HALVELLA. Decorative.

Rose pink. Large ..................... 2.50

HELEN DURNBAUGH. Hydrid Cactus.

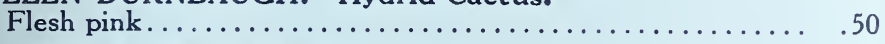

HOCHSAI. Decorative.

Yellow heavily suffused red................... 35

HORT FIET. Decorative.

Large salmon pink . . . . . . . . . 50

INSULINDE. Decorative.

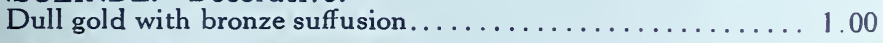

ISLAM PATROL. Decorative.

Deep red tipped gold .................... 2.50

I. W. BERNHEIM.

Large ox-blood red. Fine.................. 50

J. D. WIGGINTON. Decorative.

Tan shading lighter at center............... 1.50 
JEANE CHAZOT. Hybrid Cactus.

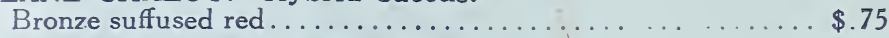

JERSEY'S BEAUTY. Decorative.

Erosine pink on long stems................. 5.00

JERSEY'S JEWELL. Decorative.

Mallow pink ...................... 3.50

JERSEY'S PRIDE. Decorative.

Apricot orange. .................... 1.50

JERSEY'S KING. Decorative.

Begonia rose. .................... 1.50

JO. BUTLER. Decorative.

Deep yellow. Very fine.................... 1.00

JOE WIGGINTON. Decorative.

Empire yellow with pinkish suffusion on outer petals...... 1.50

JUDGE A. B. PARKER. Decorative.

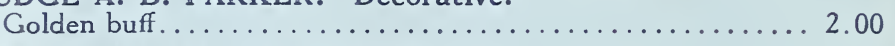

JUDGE MAREAN. Decorative.

Orange, yellow and gold ................... 1.00

J. W. DAVIES. Decorative.

Deep cerise shading lighter.................. 1.50

KALIF. Hybrid Cactus.

Brilliant pure scarlet..................... .50

KATIE BOTT. Cactus.

Wonderful free blooming scarlet. . . . . . . . . . . . . 35

KING OF SHOWS. Show.

Deep butter yellow....................... 50

KING OF COMMERCE. Decorative.

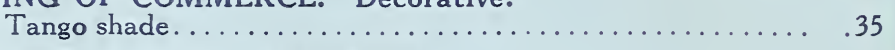

KITTIE DUNLAP. Decorative.

American Beauty Rose....................... 1.00

LADY BETTY. Decorative.

Creamy white with pink suffusion $\ldots \ldots \ldots \ldots \ldots \ldots \ldots \ldots$

LA FAVORITE. Hybrid Cactus.

Flaming orange cactus on long stiff stems........... 75

LAURINE. Decorative.

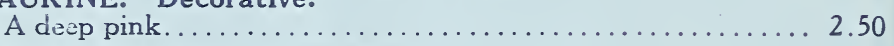

LILLY WARREN. Decorative.

Deep yellow with bronze center . . . . . . . . . . . . . 50

LOLITA VELASCO. Cactus.

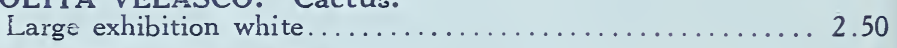

MADONNA. Decorative.

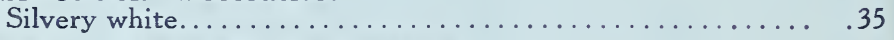

MAD. V. VASSIER. Decorative.

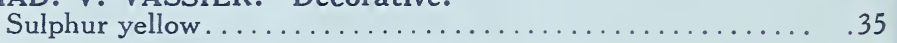

MARGARET MASSON. Decorative.

Silvery rose pink................... 5.00

MARIPOSA. Hybrid Cactus.

Delicate pink. Immense . . . . . . . . . . . . . 2.00

MAYBELLE ECKLAND. Decorative.

Dark maroon ........................... 1.00

MEPHISTOPHELES. Decorative.

Ruby red with some yellow tips. . . . . . . . . . . 1.50

MERRICK. Decorative.

Large velvety crimson.................. 3.00

MILLIONAIRE. Decorative.

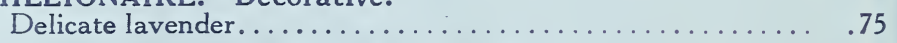

MILTON EDWARDS. Hybrid Cactus.

Herna.......................... 1.00

MISS STRANGE. Peony.

Coppery yellow suffused salmon and apricot. Wonderful

garden and exhibition dahlia. ................. 1.00

MR. CROWLEY. Decorative.

Salmon pink with golden yellow base............. 1.50

MRS. JAS. HICKMAN. Hybrid Cactus.

Deep yellow............................ 50

MRS. OSCAR FENLEY. Peony.

Ashes of roses...................... 1.00 
MRS. F. T. STREET. Cactus.

Purple tipped white.....................\$1.00

MRS. ALEC WOODRUFF. Peony.

Suffusion of copper and rose.................. 1.00

MRS. EDNA SPENCER. Cactus.

White, shading to lavender................... 50

MRS. MAZZONI, Single.

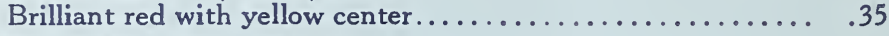

MRS. SCHLANG. Peony.

Deep orchid lavender....................... 50

MRS. CARL SALBACH. Decorative.

Wonderful mallow pink....................... 75

MRS. GEO. ELKINS, JR. Decorative.

Old gold suffused salmon...................... 1.00

MRS. E. L. LINDSEY. Decorative.

Scarlet and gold .............................. 00

MRS. JESSE SEAL. Peony.

Old rose with gold shadings.................. 50

MRS. I. deVER WARNER. Decorative.

Mauve pink............................ 1.00

MRS. JOHN SCHEEPERS. Decorative.

Clear canary yellow. Pink suffusion............... 1.50

MRS. GEO. GORDON. Peony.

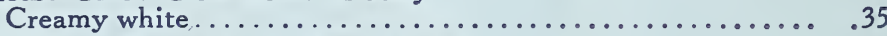

MRS. E. F. SMITH. Decorative.

Creamy white, lemon center................... 1.00

MRS. W. E. ESTES. Hybrid Cactus.

Pure white, long stems.................... .50

MT. SHASTA. Hybrid Cactus.

Light shell pink. ......................... 1.00

NANCY HELEN. Decorative.

Delicate shrimp pink........................ 75

NOBILIS. Decorative.

Red and white equally distributed . .............. 1.50

OSAM SHUDOW. Decorative.

Old rose suffused lilac...................... 2.00

PAPILLION. Hybrid Cactus.

Old rose with golden lights................. 2.50

PATRICK O'MARA. Decorative.

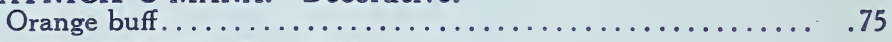

PATSY QUINN. Decorative.

Deep velvety red......................... 3.00

PAUL MICHAEL. Decorative.

Golden yellow......................... 1.00

PEARL WHITE. Decorative.

White on long stiff stems................... 1.00

PRESIDENT WILSON. Decorative.

Crimson tipped white....................... 2.00

PIERROT. Cactus.

Amber tipped white...................... 50

PIERRE GRISSARD. Decorative.

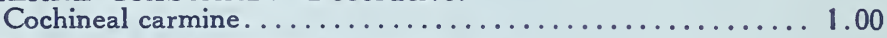

PRIDE OF CALIFORNIA. Decorative.

Deep red shaded maroon..................... 50

PRIDE OF SAN FRANCISCO. Decorative.

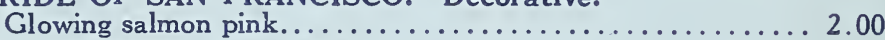

PRIZETAKER. Decorative.

Red, gold, bronze and purple................... . 75

PORTHOS. Decorative.

Rich dark red........................... 50

P. W. JANSEN. Peony.

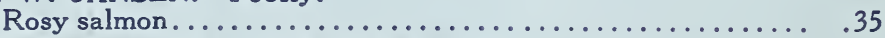

QUEEN ZENOBIA. Hybrid Cactus.

Pansy purple............................. 00

RODMAN WANAMAKER. Hybrid Cactus.

Pink shading to lighter center................ 5.00

ROOKWOOD. Decorative.

Bright cerise rose....................... 2.00

ROSA NELL. Decorative.

Clear bright rose......................... 1.00

RUSSELL HARNED. Peony.

Brilliant velvety red....................... 50

RUTH STRANGE. Peony.

Brilliant scarlet. ............... 
SHANNON. Decorative.

Big, bold golden orange. Will last a week as a cut flower.

Best commercial variety we grow...............\$2.00

SISTER ANNIE. Show.

Deep burnt orange shading to coppery yellow at tips ..... . 75

SHUDOWS LAVENDER. Decorative.

Silvery lavender........................ 2.50

SKAGERRAK. Hybrid Cactus.

Lemon yellow on long stiff stems............... 1.00

SNOWDRIFT. Decorative.

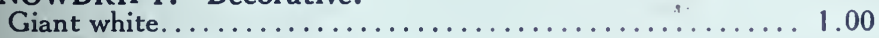

SUSAN COE. Decorative.

Rose pink suffused lilac.................... 1.50

TANTALIZER. Decorative.

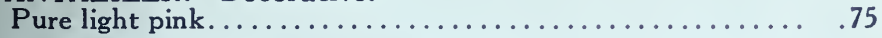

THE EMPERIOR. Decorative.

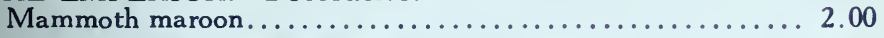

THE GRIZZLY. Decorative.

Deep velvety red........................ 1.00

THOS. OBERLIN. Cactus.

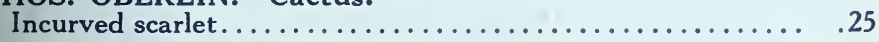

TOMMY ATKINS. Decorative.

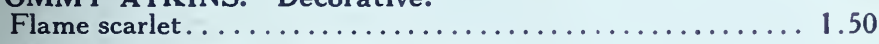

TOM WIGGINTON. Hybrid Cactus.

Canary yellow on three-foot stems.............. 1.00

U. S. A. Hybrid Cactus.

Rich glowing deep orange.

VASANTA. Hybrid Cactus.

Apricot orange with golden suffusion . . . . . . . . . 1.00

VIOLA PHILLIPS. Decorative.

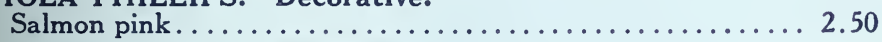

VIOLET PRICE. Decorative.

Bluish lavender. Immense. ................. 1.50

WILLIAM SLOCOMBE. Decorative.

Pure canary yellow. ........................ 1.50

YIZARD OF OZ. Decorative.

Salmon rose.............................. 00

JORLD'S BEST WHITE. Decorative.

Finest white in cultivation....................... 50

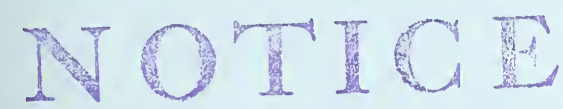

Our new farm is located on

Bardstown Road at Tyler Lane.

(OPPOSITE STRATHMOOR)

Mî todress:- Box 30, Cherokee Sta., vietille, = - Ky. 
